\title{
The effect of green tea mouthwash (Camellia sinensis) on wound healing following periodontal crown lengthening surgery; a double blind randomized controlled trial
}

\author{
Ali Forouzanfar ${ }^{1}$, Hamid Reza Arab ${ }^{1}$, Hooman Shafaee ${ }^{2 *}$, Majid Reza Mokhtari ${ }^{1}$, Shayan Golestani ${ }^{1}$ \\ ${ }^{1}$ Oral and Maxillofacial Diseases Research Center, School of Dentistry, Mashhad University of Medical Sciences, Mashhad, Iran \\ ${ }^{2}$ Department of Orthodontics, Mashhad University of Medical Sciences, Mashhad, Iran \\ Email: ${ }^{*}$ h.shafaee@gmail.com
}

Received 20 September 2012; revised 22 October 2012; accepted 28 October 2012

\begin{abstract}
Green tea has been used as a traditional medicine since $2700 \mathrm{BC}$ and several studies have shown that green tea polyphenols inhibit the growth of oral and periodontal pathogenic bacteria and can improve oral and gingival health. In this clinical study we investigated the effect of green tea mouthwash on microbial dental plaque and gingival inflammation following periodontal surgery. A total of 34 crown lengthening surgeries were included in this study. After removing periodontal dressing, green tea mouthwash for the test group and placebo for the patients in the control group were prescribed, and periodontal parameters were assessed at base line and after 2 weeks. Analysis of the data revealed the significant effect of green tea mouthwash on reducing Plaque index (PI), Gingival Index (GI) and Bleeding on probing (BOP). Therefore it would be recommended as a safe, anti-inflammatory and anti-microbial mouthwash for treating gingival inflammation and maintaining oral and gingival health.
\end{abstract}

Keywords: Green Tea; Antibacterial; Dental Plaque; Periodontal Surgery

\section{INTRODUCTION}

Tea is one of the most popular plants in the world and has been widely used for its medicinal properties. The tea plant, Camellia sinensis, is a member of the Theaceae family, and different forms of tea beverage like black, oolong, and green tea are produced from its leaves. Until recently several species and different strains of tea plants have been recognized by botanists like Camellia sinensis, Camellia assamica, Camellia pubilimba and Camellia dehungensis, however, they have similar therapeutic ef-

${ }^{*}$ Corresponding author. fects [1]. Camellia sinensis grow as small evergreen plants about 3 to 4 feet high and with a lot of branches and leaves. The leaves are dark green, oval shaped with sharp edges, and the buds are white and usually appear close together. These leaves are picked three to four times a year and are used for preparing the tea extract. In order to stop the process of tea fermentation tea leaves should be picked and heated quickly. Fermentation involves air oxidation and polymerization of major tea components that results in reducing their health benefit effects [2].

\subsection{Crown-Lengthening Procedures}

Crown-lengthening is a pre-prosthetic surgery that is performed for preparation of teeth with cervical fractures or decays to provide proper tooth preparation and adequate retention for making the restoration. This surgery facilitates impression procedures, placement of restorative margins and adapts gingival levels for esthetic results. It is important to mention that crown-lengthening surgery is done in order to preserve the biologic width of the teeth. The healthy gingival sulcus has shown an average depth of $0.69 \mathrm{~mm}$. It has been showed that the placement of a restoration within biologic width may result in gingival inflammation and consequently alveolar bone destruction. In the case of caries or tooth fracture, to ensure margin placement on sound tooth structure and retention form, the surgery should provide at least $4 \mathrm{~mm}$ from the apical extent of the caries or fracture to the alveolar bone crest. Therefore, surgical crown lengthening include the removal of soft tissue or both soft tissue and alveolar bone for designing the restoration in the accepted position without invasion through the tooth biologic width. A period of at least 6 weeks healing time should be elapsed following the surgery for the final positioning of the gingival margins. In order to promote the healing process of the gingival tissues for initiating prosthetic procedures several strategies have been pro- 
posed like local or systemic antimicrobial therapy, chemical irrigation, nutrition and diet supplements [3]. The purpose of this article is to evaluate the effect of green tea mouthwash on formation of dental plaque and the process of healing following periodontal crown lengthening surgery.

\subsection{Materials and Methods}

Thirty four patients with at least one fractured tooth, who had previously given their consent, were selected for this study. The group comprised 21 men and 13 women, with a mean age of 43 . The fractured teeth were anterior teeth or single rooted premolars and the need for the crownlengthening surgery was confirmed by a prosthodontist and a periodontist after taking a periapical radiograph using parallel technique. A local anaesthetic with vasoconstrictor (lidocaine $2 \%$ with epinephrine 1:100,000, Ultracain $^{\circledR}$, Darupakhsh, Tehran, Iran) was administered to the buccal and lingual side of the areas to be treated. A sulcular incision was made around the fractured tooth and continued for at least 2 adjacent teeth. Then a fullthickness flap was raised and the osteoplasty was done using a diamond bur. Finally the flaps were sutured and the area of the surgery was covered with periodontal dressing. Postsurgical care consisted of an antibiotic amoxicillin given orally in a dosage of $500 \mathrm{mg}$ Tid for 7 days (for reducing risk of infection) and an analgesic acetaminophen with codeine as needed for pain. After 7 days the dressing and sutures were removed. The patients divided randomly to the case and control group. For the case group (10 males and 7 females) green tea mouthwash (Green tea aqueous extract, School of Pharmacy, Mashhad University of Medical Sciences, Mashhad, Iran) was prescribed 3 times daily for 2 weeks. For the patients in the control group (11 males and 6 females) placebo was prescribed in the same manner. After 2 weeks the surgical areas were examined and the process of healing assessed using gingival index (GI), plaque index (PI) and bleeding on probing (BOP).

\subsection{Statistical Analysis}

We used the mean GI, PI, and BOP as dependent variables to reflect gingival health and healing process. Data had non-normal distribution. For comparing the two groups regarding the GI, PI, and BOP, Mann-Whitney test was applied. In all stages of evaluation, $p$ value less than 0.05 was considered significant. Data were analyzed with the use of SPSS 11.5 software (SPSS ${ }^{\circledR}$, Chicago, IL, USA).

\section{RESULTS}

A total of 34 crown lengthening surgeries were included in the present clinical study. For all patients mouthwash (green tea as the test and placebo as the control group) was prescribed, and experimental measurements were carried out after 2 weeks. Table 1 shows the data regarding clinical parameters. The differences in gingival index and bleeding on probing between two groups were significant $(\mathrm{p}<0.0001)$. Comparison of PI scores were statistically significant between the test and control groups $(\mathrm{p}<0.01)$.

\section{DISCUSSION}

The present study has clearly shown that using green tea mouthwash three times a day is endowed with significant anti-inflammatory effects (Table 1) and (Figures $\mathbf{1}$ and 2). Following periodontal surgery an antimicrobial and anti-inflammatory mouthwash is indicated for the first few postoperative weeks, particularly in crown-lengthening surgery. Over a period of more than 40 years there has been quite intense interest in the use of chemical agents to control supragingival plaque and inflammation following periodontal surgeries. The number and variation of chemical agents evaluated are quite large but most have antiseptic or antimicrobial actions. Several chemical plaque control agents have been the subject of many detailed reviews since 1980 [4-11]. Green tea is reported to have more than 4000 bioactive compounds and one third of them are categorized by polyphenols [12]. Other compounds are alkaloids (caffeine, theophylline and theobromine), amino acids, carbohydrates, proteins, chlorophyll, volatile organic compounds (chemicals that readily produce vapors and contribute to the odor of tea), fluoride, aluminum, minerals and trace elements [13]. The polyphenols, a large group of plant chemicals that includes the catechins, are thought to be

Table 1. Data regarding clinical parameters.

\begin{tabular}{cccc}
\hline & Green tea & Placebo & Comparison of two groups \\
\hline Gingival index & $0.5 \pm 0.35(0.25-1.75)$ & $1.38 \pm 0.44(0.7-2.5)$ & significant difference $(\mathrm{p}<0.0001)$ \\
Plaque index & $0.06 \pm 0.21(0-1)$ & $0.38 \pm 0.41(0-2)$ & Statistically significant difference $(\mathrm{p}<0.01)$ \\
Bleeding on probing & $13.4 \%$ & $57.4 \%$ & Significant difference $(\mathrm{p}<0.0001)$ \\
Total & 17 & 17 & 34 \\
\hline
\end{tabular}

For GI, PI and BOP values pair wise comparisons were done by Mann Whitney test with Bonferroni correction. Significant (p $<0.0001)$, statistically significant difference $(\mathrm{p}<0.01)$; mean $\pm \mathrm{SD}$, minimum-maximum values are provided in parentheses. 


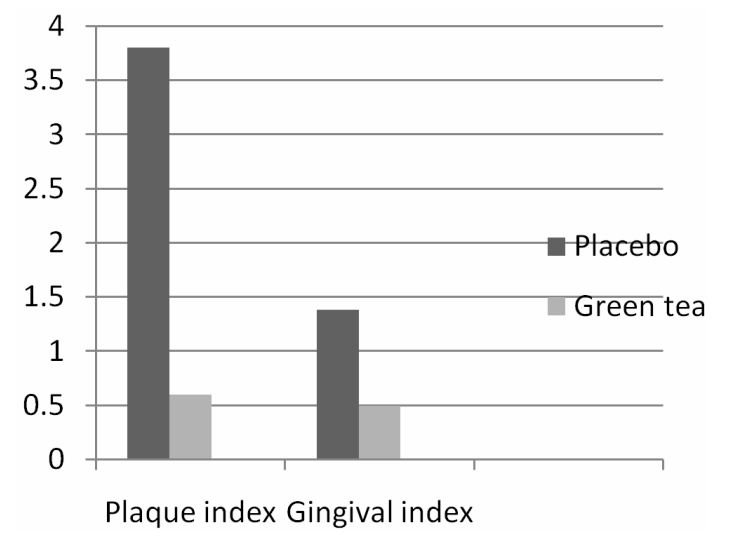

Figure 1. Graphical representations of the PI and GI scores for cases and controls.

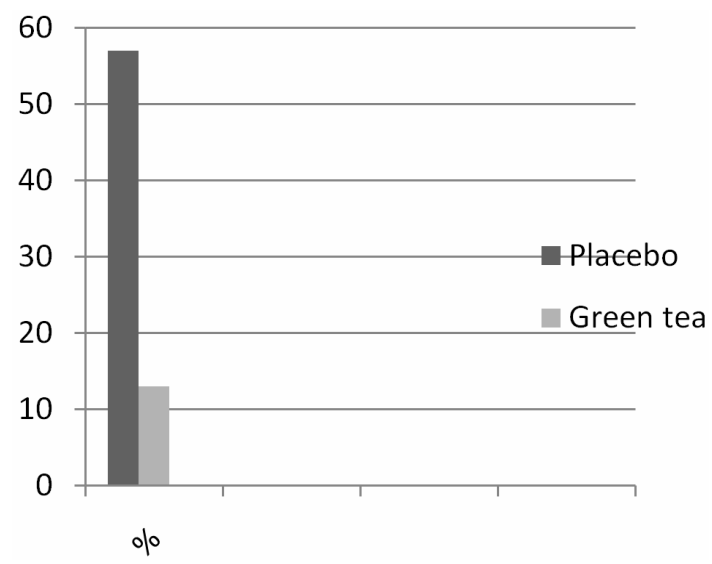

Figure 2. Graphical representations of the \% BOP for cases and controls.

responsible for the health benefits that have traditionally been attributed to tea, especially green tea [14]. Major catechins are $(-)$ epicatechin gallate (ECG), $(-)$ epicatechin (EC), (-) epigallocatechin (EGC) and (-) epigallocatechin gallate (EGCG). The most active and abundant catechin in green tea is epigallocatechin-3-gallate (EGCG) [2]. Comparison of mean plaque index between test and control groups in this clinical trial represents the inhibitory antimicrobial effects of green tea aqueous extract (Figure 1). Several in vitro studies have illustrated that green tea catechins, such as EGCG, inhibit the growth of dental plaque pathogens such as Porphyromonas gingivalis, Prevotella intermedia, and Prevotella nigrescens and the adherence of Porphyromonas gingivalis onto human buccal epithelial cells [15]. These bacteria have been strongly implicated in destruction of periodontal tissues and their reduction can lead to the improvement of periodontitis [16]. It has been demonstrated that green tea catechins that are the major tea polyphenols, inhibit the production of toxic enzymes of P. gingivalis [17]. These reports of the inhibitory effects of green tea constituents on oral pathogens may repre- sents the basis for the beneficial effect of the green tea mouthwash on gingival health after periodontal surgery. A pilot clinical study evaluated the slow-release local delivery of catechin on gingival and periodontal status. Green tea catechins put inside the hydroxypropylcellulose strips as a slow release local delivery system and inserted into pockets of periodontal patients once a week for 8 weeks. One study showed a bactericidal effect of green tea catechins against black-pigmented, Gram-negative anaerobic rods and demonstrated that the application of green tea catechin using a slow release local delivery system was effective in improving gingival inflammation [18].

In our study we used moutwash composed of extracted green tea polyphenoles which are the most therapeutic ingredient of this plant in a safe and stable formula. Following periodontal surgery, gingival inflammation versus health was assessed by gingival index and the percentage of areas with bleeding on probing. For both criteria statistical analysis demonstrated that green tea mouthwash significantly reduced inflammation for the patients in the test group. Previous studies have reported that green tea is useful for treating gingival inflammation and maintenance of oral health. An epidemiologic study showed that there is an inverse association between the daily intake of green tea and periodontal disease and suggested that drinking green tea at meals and breaks is a relatively easy habit to maintain a healthy periodontium [19]. Several other experimental and epidemiologic studies have addressed the anti-inflammatory effect of green tea supplements $[16,17]$. Physiologic studies suggested that green tea catechins can inhibit production of oxidative stress in the inflamed tissues [20,21]. Oxidative stress plays an important role in the pathogenesis of gingival inflammation, as well as many other inflammatory disorders and it is believed that antioxidants can defend against inflammatory processes [19]. Therefore, similar mechanisms might be suggested for reducing gingival inflammation after rinsing with green tea mouthwash following periodontal surgery which was specified in this clinical study.

\section{CONCLUSION}

The present study suggests that green tea aqueous extract can prevent dental plaque formation and reduce gingival inflammation following periodontal crown-lengthening surgery. Since dental plaque plays an important role in the pathogenesis of dental caries and creation of gingival inflammation, green tea mouthwash could be recommended for preventing dental caries and treating gingivitis. It could be also prescribed for patients after periodontal surgery due to its antibacterial and anti-inflammatory properties. It was also evident that green tea 
mouthwash which was used in this study had no side effects, so it would be useful and beneficial in all age groups for preventing and treating several oral and periodontal diseases and maintaining oral health.

\section{ACKNOWLEDGEMENTS}

This work was supported by a Grant of Scientific Research from the research affairs of Mashhad University of Medical Sciences, Mashhad, Iran. The authors also appreciate scientific writing centre of deputy research in Mashhad dental school for their cooperation in English writing and editing of this manuscript. The authors report no conflicts of interest related to this study.

\section{REFERENCES}

[1] Liao, S., Kao, Y.H. and Hiipakka, R.A. (2001) Green tea: Biochemical and biological basis for health benefits. $\mathrm{Vi}$ tamins and Hormones, 62, 1-94. doi:10.1016/S0083-6729(01)62001-6

[2] Arab, H., Maroofian, A., Golestani, S., Shafaee, H., Sohrabi, K. and Forouzanfar, A. (2011) Review of The therapeutic effects of Camellia sinensis (green tea) on oral and periodontal health. Journal of Medicinal Plants Research, 5, 5465-5469.

[3] Newman, M.G., Takei, H.H., Klokkevold, P. and Carranza, F.A. (2006) Carranza's Clinical Periodontology, 71, 1039-1049.

[4] Hull, P.S. (1980). Chemical inhibition of plaque. Journal of Clinical Periodontology, 7, 431-442. doi:10.1111/j.1600-051X.1980.tb02150.x

[5] Addy, M. (1986) Chlorhexidine compared with other locally delivered anti-microbials. A short review. Journal of Clinical Periodontology, 13, 957-964. doi:10.1111/j.1600-051X.1986.tb01434.x

[6] Kornman, K.S. (1986) Anti-microbial agents. In: Lee, H. and Kleinman, D.V., Eds, Dental Plaque Control Measures and Oral Hygiene Practices, IRL Press, Oxford, 121142.

[7] Mandel, I.D. (1988) Chemotherapeutic agents for controlling plaque and gingivitis. Journal of Clinical Periodontology, 15, 488-496. doi:10.1111/j.1600-051X.1988.tb01020.x

[8] Gjermo, P. (1989) Chlorhexidine and related compounds. Journal of Dental Research, 68, 1602-1608.

[9] Addy, M., Moran, J. and Wade, W. (1994) Chemical plaque control in the prevention of gingivitis and periodontitis. In: Lang, N.E. and Karring, T., Eds., Proceedings of the 71st European Work-Shop on Periodontology, Quintessence Publishing, London, 244-257.

[10] Heasman, P.A. and Seymour, RA. (1994) Pharmacologi- cal control of periodontal disease. 1. Anti-plaque agents. Journal of Dentistry, 22, 323-326.

doi:10.1016/0300-5712(94)90083-3

[11] Jackson, R.J. (1997) Metal salts, essential oils and phenols-old or new? In: Addy, M. and Moran, J.M., Eds., Toothpaste, Mouth Rinse and Other Topical Remedies in Periodontics, Periodontology, 15, 63-73.

[12] Tariq, M., Naveed, A. and Barkat Ali, K. (2010) The morphology, characteristics, and medicinal properties of Camellia sinensis' tea. Journal of Medicinal Plants Research, 4, 2028-2033.

[13] Cabrera, C., Giménez, R. and López, M.C. (2003) Determination of tea components with antioxidant activity. Journal of Agricultural and food chemistry, 51, 44274435. doi:10.1021/jf0300801

[14] Cabrera, C., Artacho, R. and Giménez, R. (2006) Beneficial effects of green tea-A review. Journal of American College of Nutrition, 25, 79-99.

[15] Makimura, M., Hirasawa, M. and Kobayashi, K. (1993) Inhibitory effect of tea catechins on collagenase activity. Journal of Periodontology, 64, 630-636. doi:10.1902/jop.1993.64.7.630

[16] Magnusson, I., Lindhe, J., Yoneyama, T. and Liljenberg, B. (1984) Recolonization of a subgingival microbiota following scaling in deep pockets. Journal of Clinical Periodontology, 11, 193-207. doi:10.1111/j.1600-051X.1984.tb01323.x

[17] Sakanaka, S. and Okada, Y. (2004) Inhibitory effects of green tea polyphenols on the production of a virulence factor of the periodontal disease-causing anaerobic bacterium Porphyromonas gingivalis. Journal of Agricultural and Food Chemistry, 52, 1688-1692. doi:10.1021/jf0302815

[18] Hirasawa, M., Takada, K., Makimura, M. and Otake, S. (2002). Improvement of periodontal status by green tea catechin using a local delivery system: A clinical pilot study. Journal of Periodontal Research, 37, 433-438. doi:10.1034/j.1600-0765.2002.01640.x

[19] Kushiyama, M., Yoshihiro, S., Masatoshi, M. and Yoshihisa, Y. (2009) Relationship between intake of green tea and periodontal disease. Journal of Periodontology, 80, 372-377. doi:10.1902/jop.2009.080510

[20] Bors, W., Heller, W., Michel, C. and Saran, M. (1990) Flavonoids as antioxidants: Determination of radicalscavenging efficiencies. Methods in Enzymology, 186, 343-355. doi:10.1016/0076-6879(90)86128-I

[21] Rizvi, S.I., Zaid, M.A., Anis, R. and Mishra, N. (2005) Protective role of tea catechins against oxidation-induced damage of type 2 diabetic erythrocytes. Clinical and EXperimental Pharmacology and Physiology, 32, 70-75. doi:10.1111/j.1440-1681.2005.04160.x 